\title{
Transición de los sistemas generalizados de preferencias: un cambio de política en cooperación internacional ${ }^{*}$
}

\author{
Recibido: 15 de abril de 2020 - Aprobado: 22 de abril de 2020 \\ https://doi.org/10.22395/seec.v23n54a4
}

José Jaime Baena Rojas*

RESUMEN

Tras el fin de la Segunda Guerra Mundial, la cooperación internacional aumentó notablemente. Este escenario propició el auge de preferencias arancelarias unilaterales a través de los sistemas generalizados de preferencias (SGP), entendidos como otro mecanismo para promover el desarrollo económico y social. De este modo, en este documento se efectúa un estudio descriptivo sobre el comportamiento de este tipo de tratados internacionales a partir de la base de datos de los acuerdos comerciales regionales de la Organización Mundial de Comercio. Se concluye que los SGP, concedidos a los países menos adelantados, parecen extinguirse a causa de los nuevos intereses de los países adelantados que firman acuerdos de libre comercio (ALC) para acabar ciertos SGP, y porque los ALC permiten crear disposiciones normativas adicionales al margen de la OMC.

\section{PALABRAS CLAVE}

Sistemas generalizados de preferencias; preferencias arancelarias unilaterales; acuerdos de libre comercio; cooperación internacional; relaciones internacionales.

\section{CLASIFICACIÓN JEL}

F53, N00, O10, P45

\section{CONTENIDO}

Introducción; 1. Los SGP como acuerdos preferenciales unilaterales dentro del sistema multilateral de comercio; 2. Metodología; 3 . Resultados y evidencias sobre la transición de los sistemas generalizados de preferencias; 4. Conclusiones; Bibliografía.

Este documento se deriva del proyecto titulado Aproximaciones a la coyuntura económica desde la multidisciplinariedad de los negocios internacionales y el comercio mundial adscrito al Grupo de Investigación Organización y Gerencia (Orygen), reconocido por Colciencias, de la Institución Universitaria Ceipa.

* Profesional en Ciencia Política, Universidad Nacional de Colombia, Medellín, Colombia. Profesional en Negocios Internacionales, Institución Universitaria Esumer, Medellín, Colombia. Especialista en Comercio Internacional, Escuela Europea de Dirección y Empresa (Eude), Barcelona, España. Magíster en Internacionalización y doctor en Derecho y Ciencia Política, Universidad de Barcelona, Barcelona, España. Profesor investigador, Institución Universitaria Ceipa, Medellín, Colombia. Correo electrónico: jose.baena@ceipa.edu.co. Orcid: https://orcid. org/0000-0002-0915-4087 


\title{
TRANSITION TO THE GENERALIZED SYSTEMS OF PREFERENCES: A CHANGE OF POLICY IN INTERNATIONAL COOPERATION
}

\begin{abstract}
After the end of World War II, the international cooperation increased notoriously, a scenario that propitiated the boom of unilateral preferential tariffs through a generalized system of preferences (GSP), understood as another mechanism for promoting the social and economic development. So, this article performs a descriptive study on the behaviour of these type of international treaties based on databases of the regional commercial agreements of the World Trade Organization. This article concludes that the GSP, conceded to the least developed countries, seem to be extinguishing given the new interest of the developed countries currently signing free trade agreements (FTA) for ending certain SGP, additionally because the FTA allows the creation of additional normative dispositions apart from the WTO.
\end{abstract}

\section{KEY WORDS}

Generalized system of preferences; unilateral preferential tariffs; free trade agreements; international cooperation; international relations.

\section{JEL CLASSIFICATION}

F53, N00, O10, P45

\section{CONTENT}

Introduction; 1. The GSP as unilateral preferential agreements within the multilateral commerce system; 2. Methodology employed in this article; 3 . Results and evidence on the transition of the generalized systems of preferences; 4 . Conclusions; Bibliography.

\section{TRANSIÇÃO DOS SISTEMAS GERALIZADOS DE PREFERÊNCIAS UMA MUDANÇA DE POLÍTICA NA COOPERAÇÃO INTERNACIONAL \\ RESUMO}

Após o final da Segunda Guerra Mundial, a cooperação internacional aumentou de forma notável, o que propiciou o auge de preferências aduaneiras unilaterais por meio do sistema geral de preferências (SGP), entendido como outro mecanismo para promover o desenvolvimento econômico e social. Desse modo, neste documento, é realizado um estudo descritivo sobre o comportamento desse tipo de tratados internacionais a partir da base de dados dos acordos comerciais regionais da Organização Mundial do Comércio (OMC). Conclui-se que os SGP, concedidos aos países menos desenvolvidos, parecem desaparecer, tendo em vista os novos interesses dos países desenvolvidos que assinam acordos de livre comércio (ACL) para acabar com alguns deles; além disso, porque os ALC permitem criar dispositivos regulamentares adicionais à margem da OMC.

\section{PALAVRAS CHAVE}

Sistema geral de preferências; preferências aduaneiras unilaterais; acordos de livre comércio; cooperação internacional; relações internacionais.

\section{CLASIFICACÇÃO JEL}

F53, N00, O10, P45

\section{CONTEÚDO}

Introdução; 1. Os SGP como acordos preferenciais unilaterais dentro do sistema multilateral de comércio; 2. Metodologia utilizada no estudo; 3 . Resultados e achados sobre a transição dos sistemas gerais de preferências; 4 . Conclusões; Bibliografia. 


\section{INTRODUCCIÓN}

De acuerdo con Estevadeordal y Suominen (2009), la evidencia empírica indica que aproximadamente la mitad del comercio mundial se efectúa entre los países que han logrado llevar a cabo tratados comerciales internacionales entre sí. Por este motivo, el presente tema se erige como un asunto trascendental de estudio debido a la dinámica del comercio internacional. Así pues, la configuración del sistema multilateral de comercio, a la cabeza del Acuerdo General sobre Aranceles Aduaneros y Comercio (GATT) y la Organización Mundial del Comercio $(\mathrm{OMC})$, ha generado un entorno más propicio para promover los intercambios de bienes y servicios entre países. De esta manera, se deja de lado la implementación de políticas proteccionistas excesivas las cuales pueden resultar perniciosas para el crecimiento del comercio en general (Baena, 2018; Vanegas y Baena, 2019; Baena y Londoño, 2020).

De este modo, sobre el tema central de este estudio, la misma OMC (2019) categoriza los tratados internacionales para liberalizar el comercio como arreglos comerciales preferenciales (ACP) que se caracterizan porque los países menos adelantados reciben preferencias comerciales unilaterales provenientes usualmente de países adelantados. Lo anterior se realiza a través de instrumentos de liberalización conocidos como sistemas generalizados de preferencias (SGP). Por otro lado, la OMC (2019) categoriza este tipo de tratados internacionales, como acuerdos comerciales regionales (ACR) caracterizados por su naturaleza recíproca entre dos o más miembros que buscan disminuir los niveles de proteccionismo conjuntamente a través de acuerdos de libre comercio (ALC) o por medio de iniciativas más complejas y ambiciosas como las integraciones económicas (IE).

Así pues, el presente artículo apunta específicamente a analizar dentro del sistema multilateral de comercio el comportamiento, en un sentido amplio, de cierto tipo de tratados comerciales. Este es el caso de los SGP que se entienden dentro de las relaciones internacionales, como un componente más para la promoción de la cooperación internacional hacia los países menos adelantados que por lo general, proviene de los países adelantados. Estos últimos buscan, claramente, generar entornos más propicios para afianzar el comercio internacional y dinamizar la economía mundial a través de la promoción del desarrollo económico y social (Tassara, 2016). Es por este motivo que Duarte y González (2014) agregan que el fin de la Segunda Guerra Mundial no solo trajo consigo la configuración de la Organización de las Naciones Unidas como elemento estabilizador para las relaciones internacionales, sino que esta institución terminó catapultando la cooperación internacional por medio de programas especiales para la transferencia directa de recursos pecuniarios 
destinados para estados con necesidades específicas, incluyendo los países menos adelantados, con el fin de mejorar ciertos indicadores sociales y de desarrollo.

Los SGP han tenido entonces un rol crucial en el devenir del comercio internacional, especialmente para los países menos adelantados, los cuales han recibido un tratamiento preferencial arancelario unilateral que ha promovido el comercio exterior de todos estos países caracterizados por ser grandes exportadores de productos agrícolas, y que pueden resultar claves para los estados otorgantes de este tipo de beneficios (OCDE, 2005). Sobre los SGP la evidencia sugiere, además, que si bien este tipo de tratados no tienen por qué representar ningún tipo de contraprestación, estos pueden generar, en determinados casos, que algunos países menos adelantados implementen medidas concretas que den un tratamiento favorable a la inversión extrajera, lo cual, si bien puede significar mayor recepción de capital, también puede suponer una potencial ventaja para las empresas procedentes de países adelantados respecto a las empresas nacionales que no poseen las mismas capacidades (Büthe y Milner, 2008).

En este sentido, este documento tiene como principal propósito evidenciar el devenir actual de los SGP dentro del sistema multilateral de comercio dejando claro la manera en que este tipo de tratados comerciales internacionales están experimentando una notable transición hacia ALC. Estos últimos en los cuales además los países signatarios pueden negociar, en un tiempo relativamente rápido, una significativa multiplicidad de temas, entre los que se destacan aquellos alusivos al comercio agrícola, subvenciones a la exportación, obstáculos no arancelarios, mejoramiento relativo a acceso al mercado de servicios, agilización de procedimientos aduaneros, mejoramiento de ciertas disciplinas en los textos jurídicos de la OMC, acuerdos sobre el medio ambiente, las propiedad intelectual, entre otros tantos temas más. De esta manera, se deja en evidencia la existencia de una gran cantidad de asuntos dentro del sistema multilateral de comercio que no se lograron resolver en la Ronda de Doha y que tampoco logran resolverse en las conferencias ministeriales de la OMC (Crawford y Laird, 2000).

Así pues, la situación anterior sobre la transición de los SGP hacia ALC parece desdibujar el panorama de cooperación internacional vía preferencias arancelarias unilaterales para los países menos adelantados, lo que deja entrever un cambio en la política internacional de algunos Estados hacia un enfoque más realista de las relaciones internacionales. Situación que evidencia nuevas dificultades de voluntad política dentro del sistema multilateral de comercio reafirmándose la idea, según Fernández (2009), de que el sistema internacional atraviesa importantes desafíos en donde deben ser superadas ciertas discrepancias entre los Estados, los cuales 
pueden percibir limitada su capacidad soberana de actuación político-económica dentro del paradigma de gobierno mundial.

Así, el presente artículo plantea una discusión respecto a los motivos por los cuales el ideal de la cooperación internacional, vía preferencias arancelarias unilaterales, parece perder notoriedad dadas las dificultades de consenso dentro de la OMC. Según Rosales (2009), estas dificultades se explican por los aún visibles efectos de la gran recesión que parecen afectar este tipo de iniciativas de apoyo para los países menos adelantados. Por otra parte, Baena, Montoya y Torres (2017), apuntan que estas dificultades son producto de incertidumbre con respecto a los posibles episodios de intensificación de proteccionismo dentro de la política de comercio exterior de los países.

Dicho todo lo anterior, el presente documento procede a explicar el apartado metodológico. En este sentido, se expone el tipo de estudio aplicado dentro de esta investigación y se señalan las fuentes oficiales consultadas derivadas de Acuerdos Comerciales Regionales Base de Datos como asimismo la Base de datos sobre los arreglos comerciales preferenciales de la OMC. Posteriormente, se presenta el apartado de los resultados. Por medio de gráficos y tablas se explica, a nivel descriptivo, el comportamiento de los tratados comerciales internacionales objeto de estudio. Lo anterior se hace con el fin de validar el planteamiento principal de esta investigación sobre la transición de los SGP a ALC dentro del sistema multilateral de comercio y su impacto para la cooperación internacional. Finalmente, se ofrecen las conclusiones y se presentan las posibles futuras líneas de trabajo derivadas de toda esta aproximación teórica.

\section{LOS SGP COMO ACUERDOS PREFERENCIALES UNILATERALES DENTRO DEL SISTEMA MULTILATERAL DE COMERCIO}

Si bien los tratados comerciales internacionales no son nuevos, su influencia dentro del comercio internacional parece tomar, hasta ahora, un protagonismo distintivo a causa de sus efectos de contención sobre el proteccionismo. Así, los tratados comerciales constituyen en la actualidad un instrumento esencial para disminuir las políticas restrictivas de los Estados, reflejadas en el uso desmedido de las barreras arancelarias y las barreras no arancelarias, además de promover la facilitación de acceso a mercados con el propósito de impulsar el desarrollo y el crecimiento económico de los países (Lynch, 2010).

Cabe agregar que el protagonismo de los tratados comerciales internacionales deviene, no solo del hecho de cómo estos promueven la liberalización del comercio internacional para constituirse en un elemento estratégico para la inserción de 
cualquier país en la economía internacional (Baena y Fernández, 2016; Baena, 2019), sino que, incluso, dicho protagonismo deviene del hecho de que los tratados comerciales se han convertido en un trascendental e innovador instrumento para la creación de ordenamientos jurídicos en todos los países que forman parte de estos esquemas de intercambio (Trejos, 2009).

En este sentido, todos estos tratados pueden, en general, favorecer las relaciones internacionales y considerar las posibilidades que estos ofrecen para hacer más estrechos los lazos comerciales e, incluso, políticos. Precisamente, su proceso de negociación no solo se suele enfocar en el intercambio de productos para promover el desarrollo social, sino también para afianzar los vínculos de confianza y consenso entre los países signatarios (Bearce, 2003; Milewicz, Hollway, Peacock, y Snidal, 2018).

Sobre los tratados comerciales internacionales, concebidos como acuerdos entre Estados para la disminución de barreras arancelarias y barreras no arancelarias, es preciso agregar que estos, pueden agruparse en dos categorías dependiendo de sus características en torno a su propio grado de reciprocidad (véase el gráfico 1). De un lado se pueden identificar los SGP como ACP en donde existe un Estado que recibe preferencias arancelarias sin contraprestación alguna, y un Estado otorgante que, tan solo en teoría, busca incentivar la cooperación internacional. De otro lado, se pueden identificar los ALC y las IE como ACR. En el primer caso, dos Estados se otorgan conjuntamente preferencias arancelarias con fines exclusivamente comerciales. En el segundo caso, tres o más Estados se otorgan conjuntamente, no solo preferencias arancelarias, sino que también buscan alcanzar mancomunadamente una serie de objetivos económicos, sociales y políticos (Kembayev, 2009; OMC, 2011; Missios, Saggi, y Yildiz, 2016).

De esta manera, cabe anotar que la literatura científica evidencia una amplia y diversa gama de terminología técnica para referirse a los tratados comerciales internacionales que, a nivel genérico, pueden ser entendidos como unilaterales, bilaterales o multilaterales, pero que comparten en esencia el mismo interés por liberalizar el comercio (Rivera-Batiz y Oliva, 2003; Mansfield y Milner, 2012).

Según Giordano, Mesquita y Quevedo (2004), los SGP, como tratados comerciales internacionales, poseen una característica distintiva en relación con los demás tipos de tratados: dentro de estos, desde la configuración del GATT en 1947, los países han venido interactuando bajo una premisa de liberalización comercial asimétrica que se evidencia en una serie de beneficios y preferencias arancelarias no recíprocas exclusivamente a favor de los países menos adelantados dadas sus condiciones particulares de desarrollo y crecimiento económico. Esta situación se fundamenta, desde los orígenes del sistema multilateral de comercio, en las 
disposiciones del trato especial y diferenciado (TED), conocida como una medida especial que otorga a los países menos adelantados una serie de ayudas, beneficios o ventajas excepcionales que representan una flexibilización dentro del ordenamiento jurídico. Todo esto se hace a través del reconocimiento explícito de las diferencias económicas entre los países. De este modo, el TED constituye, dentro del actual sistema de comercio internacional, toda una herramienta para el fomento de normas específicas que garanticen una mayor igualdad y que pondere la interacción económica entre los países (García y Arévalo, 2014).

\section{Gráfico 1. Tipología y características de los tratados comerciales dentro de la OMC}
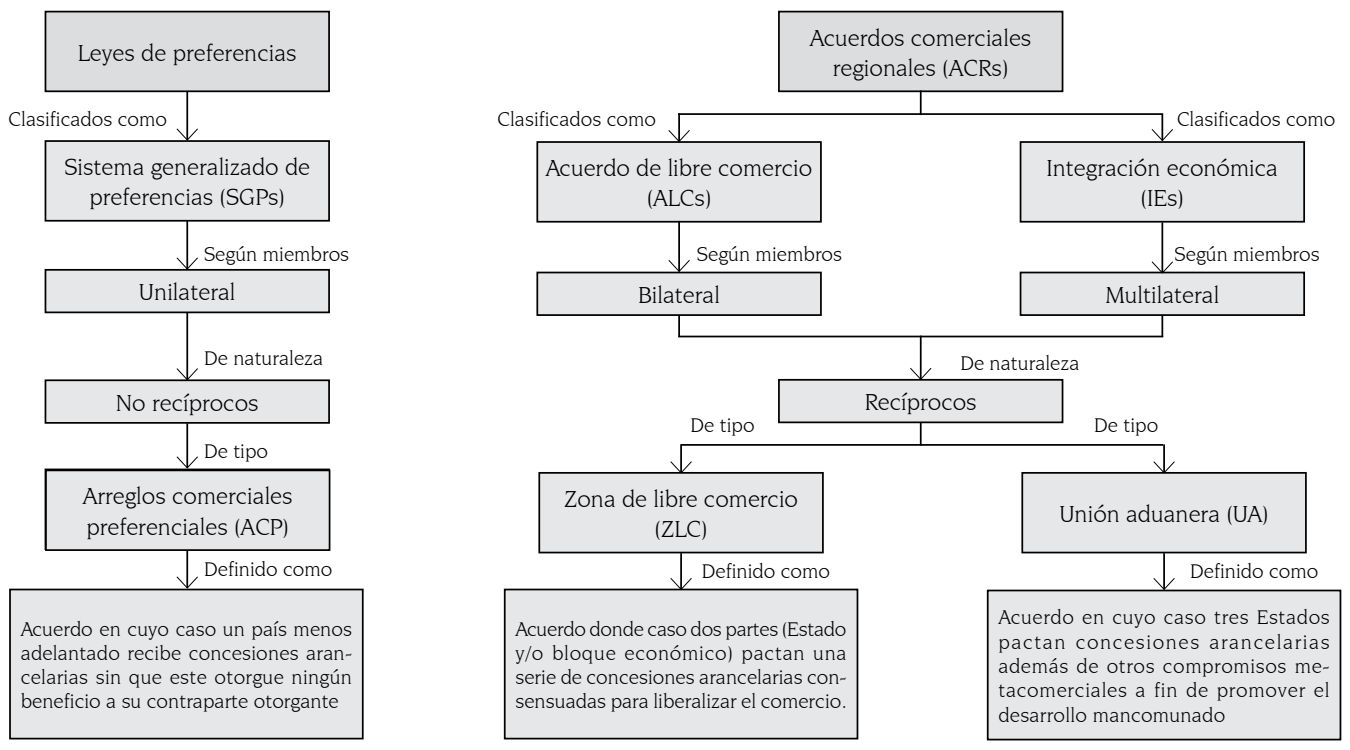

Fuente: elaboración propia a partir de Kemp y Wan (1976); Kolb (2008) y OMC (2019).

Según Díez (2001), el principio del TED se encuentra consignado dentro del artículo XVIII, "Ayuda del Estado para favorecer el desarrollo económico", del GATT. Este artículo no solo considera el desarrollo dispar de los países dentro del comercio internacional, sino que se torna como un importante elemento de partida para la firma de SGP, dado que este afianza la negociación de tratados que otorguen concesiones arancelarias no recíprocas con el objetivo de amparar los países menos adelantados los cuales suelen evidenciar inconvenientes en su desarrollo y crecimiento económico. Por otro lado, el artículo XXIV "Aplicación territorial. Tráfico fronterizo, uniones aduaneras y zonas de libre comercio" del GATT, es otro importante punto de partida para la firma de tratados internacionales comerciales como ALC e incluso de IE. Pero paradójicamente, con el tiempo y la firma de diversos nuevos 
tratados, todos estos terminaron generando fuertes debates en torno a su validez, teniendo en cuenta el artículo I "Trato general de la nación más favorecida" del GATT parecía generar incompatibilidades que no se podían pasar por alto (Lacarte y Granados, 2004; Kennedy, 2012; Cardona, 2015).

Ahora bien en cualquier caso, todo este episodio se logró esclarecer tras el desarrollo de las diversas rondas de negociación del mismo GATT. A través de estas se alcanzaron contribuciones esenciales para la creación de decisiones y nuevas disposiciones normativas expresadas en los textos jurídicos que complementan, en la actualidad, las normas de la OMC. La Ronda de Tokio de 1973, por ejemplo, dio origen a la conocida cláusula de habilitación que hizo posible en definitiva llevar a cabo tratados comerciales internacionales con el objeto de suprimir trabas al comercio, tales como barreras arancelarias y no arancelarias. Con ello, se excluyó excepcionalmente el principio de la nación más favorecida consignado en el artículo I del GATT (Huici, 2006; De la Reza, 2015; Cardona, 2017).

Al retomar la idea sobre la liberalización asimétrica de los SGP, cabe agregar que esta condición hace que este tipo de tratados sean comprendidos como un componente de la cooperación internacional gracias a la discrecionalidad en el otorgamiento de las preferencias arancelarias por parte de ciertos países adelantados sobre los países menos adelantados, mientras que los tratados comerciales ALC y las IE apuntan a otros propósitos diferentes, no necesariamente en la misma línea de la cooperación internacional (Zahrnt, 2005).

Así entonces, sobre los tratados internacionales es preciso señalar que estos se encuentran condicionados particularmente por un escenario de heterogeneidad que a su vez caracteriza las relaciones internacionales. De ahí que los SGP puedan ser comprendidos como un instrumento de cooperación considerando que ciertos países buscan beneficiar a otros sin un interés particular (Trottier, 1987; Scott, 2016). De acuerdo con Cassimatis (2011), los SGP históricamente han permitido incentivar el crecimiento y desarrollo social dentro del marco de la cooperación internacional debido a que estos tratados han sido percibidos como una herramienta para lograr que los países menos adelantados, en su condición de beneficiarios, adapten dentro de sus economías ciertas buenas prácticas propias de los países adelantados, en condición de otorgantes, todo lo que respecta a la implementación de derechos sociales, civiles y laborales.

Sin embargo, según Jones (2006), la discrecionalidad en el otorgamiento de las preferencias arancelarias conlleva a la no renovación de los SGP y, por consiguiente, a que estos experimenten una transición hacia ALC. Por medio de estos los países 
adelantados o algunos que no lo son y otorgan estos beneficios, dejan de conceder tratados en donde no existe reciprocidad alguna y en donde, logran llevar a cabo acuerdos más atractivos que también les otorgan acceso a los mercados de sus antiguos beneficiarios. Además, estos tratados permiten concertar normas puntuales que no se logran dentro de la OMC debido a los múltiples problemas de consenso dentro de la Ronda de Doha.

De hecho, Hartman (2013) afirma que el fracaso de Doha y los problemas en el marco de las negociaciones dentro de la OMC están directamente relacionados con la proliferación de nuevos ALC. Esto se debe a que los países adelantados mantienen una postura férrea de no continuar liberalizando su comercio exterior en las circunstancias actuales del sistema multilateral de comercio en donde temas como el comercio agrícola, los servicios, los procedimientos aduaneros, la creación de nuevos textos jurídicos, entre otros tantos, han generado una significativa ruptura en las conversaciones entre los países adelantados y los menos adelantados.

En esta misma línea, Dos Santos, Farias y da Cunha (2005) agregan que esta falta de consenso y voluntad política entre países adelantados y países menos adelantados dentro de la OMC sugiere un escenario pesimista para la consecución del éxito dentro de las negociaciones comerciales en el mediano y largo plazo. Todo esto se considera teniendo en cuenta de que estos últimos países, si bien son mayoría dentro del sistema multilateral de comercio, carecen en cualquier caso de capacidad suficiente para generar una mayor influencia dentro del modelo actual de relaciones internacionales.

Aparte de toda esta situación sobre los problemas de institucionalidad dentro de la OMC, es necesario agregar que el comportamiento de la economía mundial tampoco ha logrado reencausarse a plenitud tras la debacle de la gran recesión del 2008 y 2009, lo cual afecta toda la dinámica de la cooperación internacional (Gurtner, 2010; Robinson, 2012). En este sentido, los países adelantados han venido restringiendo sus flujos de capital en un intento por amparar sus economías. No obstante, todas estas medidas parecen generar contradictoriamente una serie de efectos perjudiciales para el crecimiento del comercio internacional (Acharya y Bengui, 2018).

De acuerdo con lo anterior, tanto los problemas de institucionalidad de la OMC como los efectos aún visibles de la gran recesión parecen empujar a los países adelantados - o aquellos que no lo son y otorgaban estos beneficios-, a replantear sus agendas políticas de comercio exterior. Por un lado, es necesario implementar nuevas reformas a nivel jurídico que regulen ciertos asuntos dentro del sistema multilateral de comercio. Por otro lado, los SGP y la concesión unilateral de preferencias arancelarias 
comportan un costo económico importante. Por consiguiente, estos argumentos parecen demostrar porque los SGP tienden a experimentar una notable transición hacia ALC, lo cual parece además quebrantar el ideal de cooperación internacional dentro de las relaciones internacionales (Bartels, 2003; Van der Berg, 2017).

De esta manera, las circunstancias han terminado convirtiendo, de cierto modo, los SGP en un nuevo artífice de una considerable cantidad de ALC, los cuales han experimentado en las últimas décadas un significativo aumento por diversos motivos entre los que sobresalen los dos siguientes. En primera instancia, se encuentra la multiplicidad de posibilidades que este tipo de tratados comerciales ofrecen para que sus países signatarios encuentren soluciones al margen de la OMC dado su actual estancamiento en las negociaciones de las conferencias ministeriales y particularmente en la Ronda de Doha (Urata, 2002; Crawford y Laird, 2001). En segunda instancia, se encuentran los retos que comporta precisamente participar del sistema multilateral de comercio como miembro de pleno derecho, si se considera el componente de la supranacionalidad que limita la autonomía de los Estados y que parece un poco más fácil de sobrellevar con ALC (Cardona, 2018).

En cualquier caso, todo este panorama sobre la participación de los Estados dentro del sistema multilateral de comercio por un lado y la participación de los países firmando tratados comerciales por el otro, tiende cada vez más a generar preocupación debido a que los países firmantes de ALC son, a su vez, miembros de pleno derecho de la OMC, lo que ocasiona importantes incongruencias jurídicas. Esto se debe a que los países que hacen parte de disputas comerciales en el seno de la OMC están invocando normas especiales desarrolladas dentro de estos tratados internacionales para justificar sus políticas comerciales; en muchos casos estas normas no coinciden con el GATT y los textos jurídicos (Henckels, 2008).

En definitiva, es posible cerrar la reflexión de este apartado agregando que, según Milewicz, Hollway, Peacock y Snidal (2018), si bien los tratados comerciales tienen como propósito, desde su esencia misma, ahondar en compromisos alusivos al intercambio de productos, indirectamente dentro de estos se terminan involucrando otros asuntos dada la dinámica de las relaciones internacionales. En otras palabras, los acuerdos entre los países tienden a darse no solo por intereses económicos, sino que también se pueden inmiscuir otros asuntos, tales como los derechos humanos, democracia, medio ambiente, corrupción y normas laborales. En relación con estos asuntos, las partes, por lo general, deben de tener algún tipo similitud desde su propia cosmovisión política. 


\section{METODOLOGÍA}

Dentro de este apartado se destaca la realización de una revisión de la literatura titulada Los SGP como acuerdos preferenciales unilaterales dentro del sistema multilateral de comercio en donde fueron rastreadas una serie de investigaciones reflejadas en artículos, libros, sitios web que hicieran posible identificar los atributos esenciales en torno a los sistemas generalizados de preferencias (SGP) dentro del comercio internacional. Se enfatizaron igualmente en torno a las disposiciones del Acuerdo General sobre Aranceles Aduaneros y Comercio (GATT) y se tuvieron en cuenta otros textos jurídicos complementarios de la Organización Mundial del Comercio (OMC) que aportaran luces en torno a la implementación de este tipo de tratados internacionales.

Posteriormente, tras ser completada la revisión de la literatura, se procede a analizar el comportamiento actual de los SGP para demostrar que efectivamente este tipo de acuerdos están experimentado una transición hacia ALC dado que los países adelantados y los menos adelantados están buscando nuevas alternativas para liberalizar el comercio. Además, esperan crear normas para regular ciertos aspectos comerciales que dentro del sistema multilateral de comercio no se están logrando alcanzar, dados los inconvenientes en la negociación de nuevos temas en las conferencias ministeriales y a las dificultades de consenso tal y como se evidenció en la Ronda de Doha en el seno de la OMC. En este sentido, el presente trabajo constituye un estudio descriptivo que apunta a poner en evidencia si, en efecto, los SGP están mutando a ALC y, particularmente, si toda esta situación afecta colateralmente la cooperación internacional debido a la no reciprocidad de los SGP que, en principio, fueron creados para impulsar el desarrollo económico en los países menos adelantados.

De esta manera, para lograr el presente estudio descriptivo, es imprescindible acudir al sitio web oficial de la OMC en el apartado Acuerdos Comercio Regionales Base de Datos y también considerar igualmente el apartado Base de datos sobre los arreglos comerciales preferenciales. Todo lo anterior con el propósito de lograr describir la situación actual de los SGP respecto a los ALC.

En este sentido, la presente metodología, supeditada a un estudio descriptivo, propende desde una perspectiva amplia, según Ruiz (2012), Nayar y Stanley (2015), descubrir y caracterizar aspectos nuevos de un fenómeno previamente estudiado. Un estudio descriptivo se sitúa sobre una base de conocimientos más sólida que los exploratorios (gráfico 2), ya que el problema surge de una base de conocimiento previamente establecida y conocida. Pero si se busca generar alguna hipótesis sobre 
las variables a considerarse, es necesaria una descripción completa y profunda del problema que se pretende analizar (Gómez, 2006).

Finalmente, se exponen los resultados en los que se incluye un análisis detallado a partir de los datos y tendencias arrojados por la presente investigación sobre acuerdos comerciales regionales y cooperación dentro del marco de las relaciones internacionales.

Gráfico 2. Representación del estudio descriptivo según la presente investigación

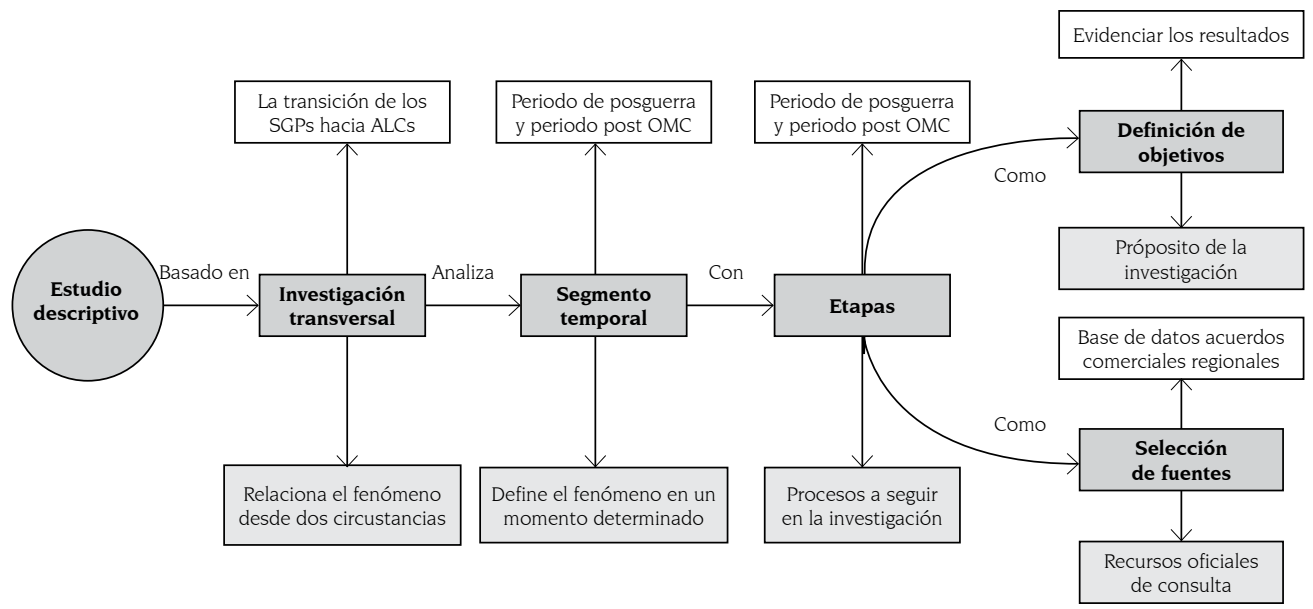

Fuente: elaboración propia basado en Del Río (2011); Ruiz (2012); Nayar y Stanley (2015).

\section{RESULTADOS Y EVIDENCIAS SOBRE LA TRANSICIÓN DE LOS SISTEMAS GENERALIZADOS DE PREFERENCIAS}

Luego de ser completada toda la reflexión previa en la revisión de la literatura en torno a los aspectos generales sobre los SGP, como acuerdos preferenciales unilaterales dentro del sistema multilateral de comercio, se procede a evidenciar el devenir actual de este tipo de tratados comerciales internacionales. Lo anterior, con el objetivo de demostrar la transición que todos estos experimentan hacia ALC y que afecta la cooperación dentro del paradigma actual de las relaciones internacionales.

Así pues, es necesario tomar como punto de partida la relación total de los SGP existentes hasta la fecha (véase la tabla 1), teniendo en cuenta que, según la base de datos de la OMC, estos iniciaron justo un año después de la firma del GATT. 
Tabla 1. Relación total de SGP desde la creación del GATT hasta la fecha

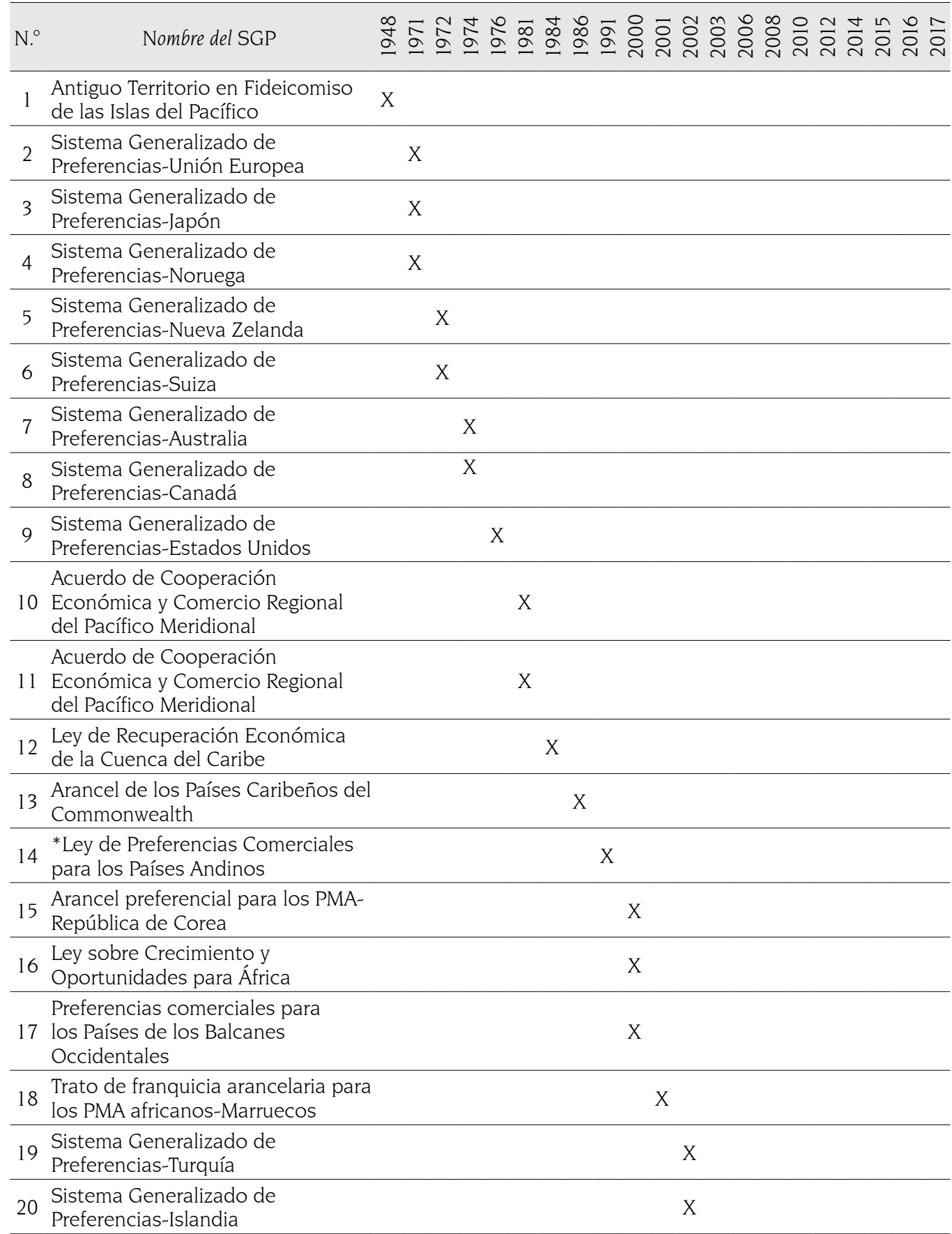




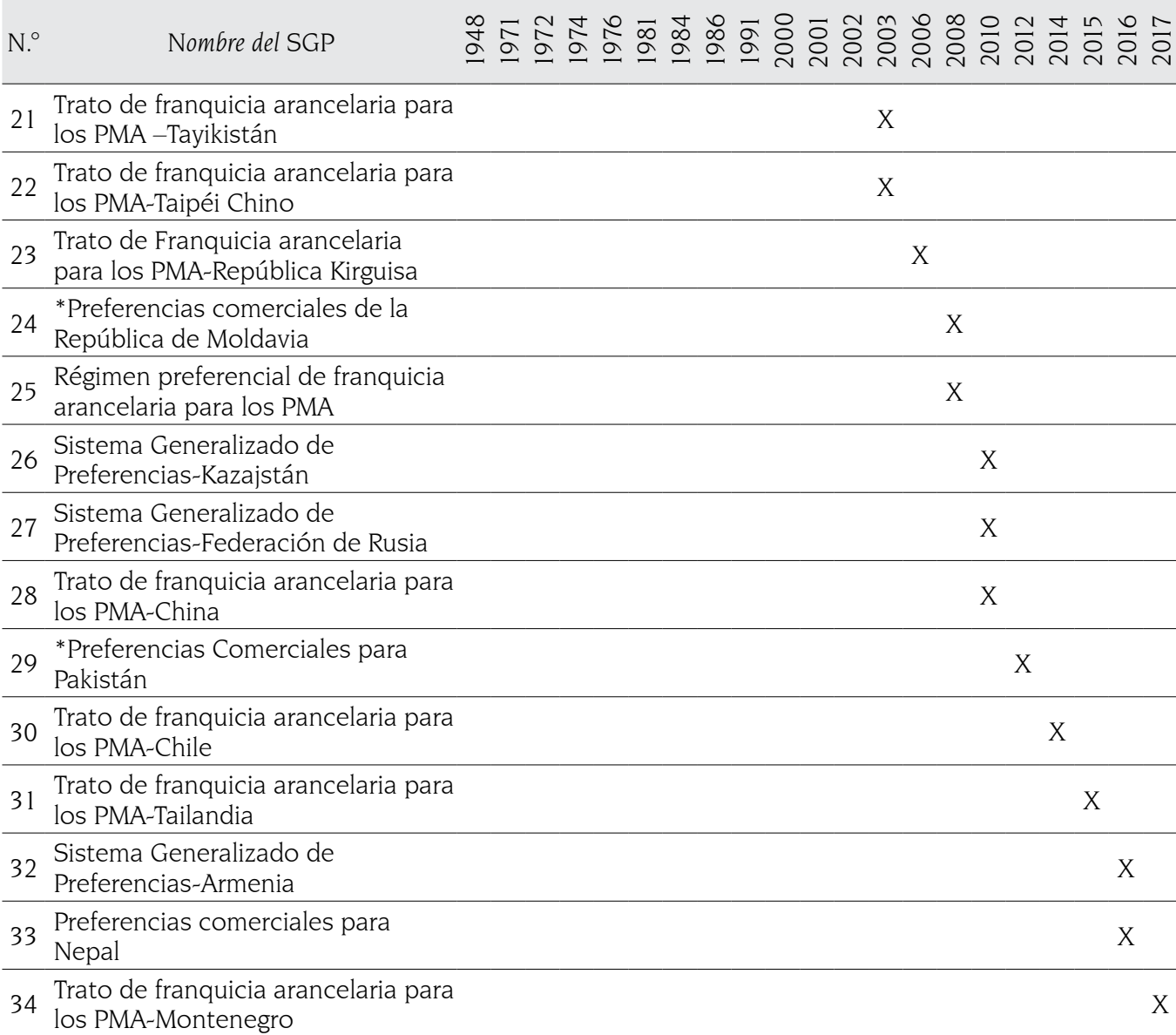

*SGP no renovado por el país otorgante.

Fuente: elaboración propia a partir de la OMC (2019a).

Según lo anterior, se lograron identificar treinta y cuatro SGP de los cuales tres se encuentran hoy vencidos. Por este motivo, se puede decir que en el presente solo treinta y un acuerdos preferenciales unilaterales continúan en vigor hasta que los países adelantados o en proceso otorgantes así lo decidan, todo esto dependiendo de las circunstancias que se presenten en los próximos años.

En consecuencia, es preciso relacionar el total de países menos adelantados beneficiarios con SGP (véase la tabla 2). Además, se incluyen los tratados de este tipo, vencidos hasta la fecha, dado que precisamente algunos de estos terminaron convirtiéndose en ALC. 


\section{Tabla 2. Los SGP por país otorgante y países beneficiarios totales}

\begin{tabular}{|c|c|c|c|}
\hline N. ${ }^{\circ}$ & Nombre del SGP & $\begin{array}{l}\mathrm{N} .{ }^{\circ} \text { de } \\
\text { beneficiarios }\end{array}$ & País otorgante \\
\hline 1 & Sistema Generalizado de Preferencias-Australia & 177 & \multirow{3}{*}{ Australia } \\
\hline \multirow[t]{2}{*}{2} & $\begin{array}{l}\text { Acuerdo de Cooperación Económica y Comercio Regional del } \\
\text { Pacífico Meridional }\end{array}$ & 13 & \\
\hline & Total & 190 & \\
\hline 3 & Antiguo Territorio en Fideicomiso de las Islas del Pacífico & 4 & \multirow{7}{*}{$\begin{array}{l}\text { Estados } \\
\text { Unidos }\end{array}$} \\
\hline 4 & Sistema Generalizado de Preferencias-Estados Unidos & 120 & \\
\hline 5 & Ley de Recuperación Económica de la Cuenca del Caribe & 17 & \\
\hline 6 & Ley de Preferencias Comerciales para los Países Andinos & 1 & \\
\hline 7 & Ley sobre Crecimiento y Oportunidades para África & 40 & \\
\hline \multirow{2}{*}{8} & Preferencias comerciales para Nepal & 1 & \\
\hline & Total & 183 & \\
\hline 9 & Sistema Generalizado de Preferencias-Nueva Zelanda & 141 & \multirow{3}{*}{$\begin{array}{l}\text { Nueva } \\
\text { Zelanda }\end{array}$} \\
\hline \multirow[t]{2}{*}{10} & $\begin{array}{l}\text { Acuerdo de Cooperación Económica y Comercio Regional del } \\
\text { Pacífico Meridional }\end{array}$ & 13 & \\
\hline & Total & 154 & \\
\hline 11 & Sistema Generalizado de Preferencias-Federación de Rusia & 154 & Rusia \\
\hline 12 & Sistema Generalizado de Preferencias-Turquía & 153 & Turquía \\
\hline 13 & Sistema Generalizado de Preferencias-Kazajistán & 153 & Kazajistán \\
\hline 14 & Sistema Generalizado de Preferencias-Armenia & 151 & Armenia \\
\hline 15 & Sistema Generalizado de Preferencias-Japón & 133 & Japón \\
\hline 16 & Sistema Generalizado de Preferencias-Canadá & 104 & \multirow{3}{*}{ Canadá } \\
\hline \multirow{2}{*}{17} & Arancel de los Países Caribeños del Commonwealth & 18 & \\
\hline & Total & 122 & \\
\hline 18 & Sistema Generalizado de Preferencias-Suiza & 123 & Suiza \\
\hline 19 & Sistema Generalizado de Preferencias-Unión Europea & 88 & \multirow{5}{*}{$\begin{array}{l}\text { Unión } \\
\text { Europea }\end{array}$} \\
\hline 20 & $\begin{array}{l}\text { Preferencias comerciales para los Países de los Balcanes Occiden- } \\
\text { tales }\end{array}$ & 6 & \\
\hline 21 & Preferencias comerciales de la República de Moldavia & 1 & \\
\hline \multirow{2}{*}{22} & Preferencias comerciales para Pakistán & 1 & \\
\hline & Total & 96 & \\
\hline 23 & Sistema Generalizado de Preferencias-Noruega & 83 & Noruega \\
\hline 24 & Trato de franquicia arancelaria para los PMA-Kirguistán & 49 & Kirguistán \\
\hline 25 & Arancel preferencial para los PMA-República de Corea & 48 & Corea del Sur \\
\hline 26 & Sistema Generalizado de Preferencias-Islandia & 48 & Islandia \\
\hline 27 & Régimen preferencial de franquicia arancelaria para los PMA & 48 & India \\
\hline
\end{tabular}




\begin{tabular}{llccc}
\hline N. & \multicolumn{1}{c}{ Nombre del SGP } & $\begin{array}{c}\text { N. }{ }^{\circ} \text { de } \\
\text { beneficiarios }\end{array}$ & País otorgante \\
\hline 26 & Trato de franquicia arancelaria para los PMA-Chile & 48 & Chile \\
\hline 29 & Trato de franquicia arancelaria para los PMA-Montenegro & 48 & Montenegro \\
\hline 30 & Trato de franquicia arancelaria para los PMA-Taipéi Chino & 47 & Taipéi Chino \\
\hline 31 & Trato de franquicia arancelaria para los PMA-Tailandia & 47 & Tailandia \\
\hline 32 & Trato de franquicia arancelaria para los PMA-Tayikistán & 45 & Tayikistán \\
\hline 33 & Trato de franquicia arancelaria para los PMA-China & 38 & China \\
\hline 34 & Trato de franquicia arancelaria para los PMA Africanos-Marruecos & 34 & Marruecos \\
\hline
\end{tabular}

Fuente: elaboración propia con datos de la OMC (2019a).

De acuerdo con los resultados previos, se puede afirmar que el mayor otorgante de preferencias arancelarias unilaterales es Australia a través de dos SGP con ciento noventa países beneficiarios; posteriormente aparece en el segundo lugar Estados Unidos a través de seis SGP con ciento ochenta y tres países beneficiarios. En la tercera posición a través de dos SGP Nueva Zelanda con un total de ciento cincuenta y cuatro países beneficiarios, todo lo anterior, entre un total de veintitrés países, en su mayoría, adelantados otorgantes de este tipo de tratados o concesiones arancelarias unilaterales.

Para el caso de los siguientes resultados, también es necesario indicar los SGP que otorgan las más altas concesiones arancelarias (véase el gráfico 3), es decir, mayores niveles de liberalización unilateral en términos porcentuales sobre las importaciones. Este aspecto facilita, sin duda, el acceso facilita, a los mercados, por lo general, de países adelantados.

De acuerdo con el gráfico 3, es posible mostrar que, de todos los países otorgantes de preferencias arancelarias unilaterales destacan, en primer lugar Chile, en segundo lugar India y en tercer lugar China, todo estos por alcanzar niveles de liberalización que superan el $85 \%$ en un total de veintitrés países, adelantados o en proceso, otorgantes de SGP. Aunque, es imprescindible señalar que este tipo de concesiones arancelarias unilaterales no siempre tienen que ser generadas exclusivamente por países adelantados, como se evidencia con los casos de India y China, entre otros, por lo cual, todo dependerá de la política de cooperación internacional de cada Estado. 


\section{Gráfico 3. SGP con mayores niveles de liberalización unilateral en su comercio para los países} beneficiarios

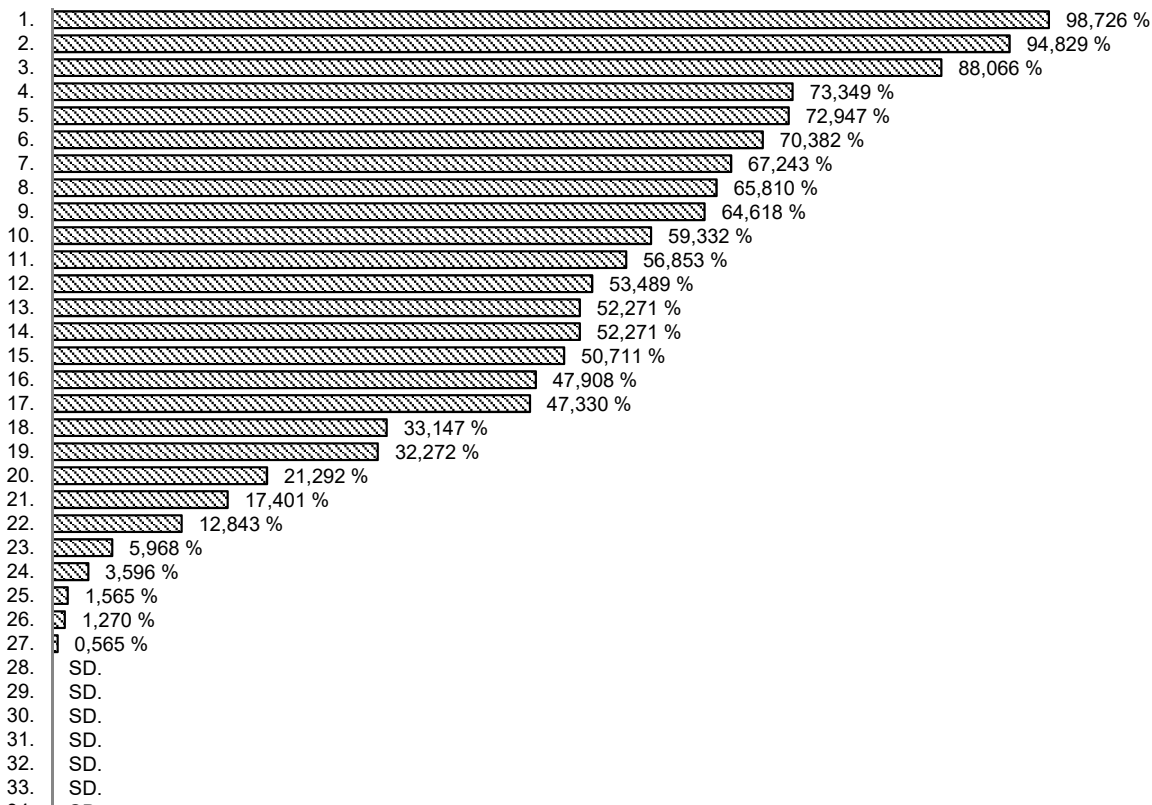

1. Trato de franquicia arancelaria para los PMA - Chile

2. Régimen preferencial de franquicia arancelaria para los PMA - India

18. Sistema Generalizado de Preferencias - Japón

19. Sistema Generalizado de Preferencias - Estados Unidos

20. Sistema Generalizado de Preferencias - Rusia

4. Arancel preferencial para los PMA - Corea del Sur

5. Preferencias comerciales de la República de Moldavia - Unión Europea

22. Sistema

22. Sistema Generalizado de Preferencias - Noruega

23. Sistema Generalizado de Preferencias - Nueva Z

24. Sistema Generalizado de Preferencias - Islandia

7. Sistema Generalizado de Preferencias - Armenia

8. Sistema Generalizado de Preferencias - Suiza

9. Sistema Generalizado de Preferencias - Unión Europea

10. Ley sobre Crecimiento y Oportunidades para África - Estados Unidos

11. Sistema Generalizado de Preferencias - Turquia

12. Trato de franquicia arancelaria para los PMA - Kirguistán

13. Acuerdo de Cooperación Económica y Comercio Regional del Pacifico Meridional -

Australia

25. Trato de franquicia arancelaria para los PMA Africanos -

Marruecos

27. Preferencias comerciales para Pakistán

28. Antiguo Territorio en Fideicomiso de las Islas del Pacífico

29. Preferencias comerciales para Nepal - Unión Europea

30. Sistema Generalizado de Preferencias - Kazajistán

14. Acuerdo de Cooperación Económica y Comercio Regional del Pacífico Meridional Nueva Zelanda

31. Trato de franquicia arancelaria para los PMA - Montenegro

32. Trato de franquicia arancelaria para los PMA - Tailandia

33. Trato de franquicia arancelaria para los PMA - Tayikistán

15. Sistema Generalizado de Preferencias - Australia

16. Ley de Preferencias Comerciales para los Países Andinos - Estados Unidos
17. Ley de Recuperación Económica de la Cuenca del Caribe - Estados Unidos

34. Preferencias comerciales para los paises de los Balcanes Occidentales Unión Europea

Fuente: elaboración propia con datos de la OMC (2019a).

En los resultados siguientes es categórico exponer los SGP que han terminado experimentado una transición a ALC (gráfico 4), ya que precisamente este hecho deja claro que la cooperación atraviesa un momento a tener en cuenta en el ámbito del comercio internacional. En este sentido, se considera la desaparición de este tipo de tratados no recíprocos. 


\section{Gráfico 4. SGP que han experimenta transición en ALC}

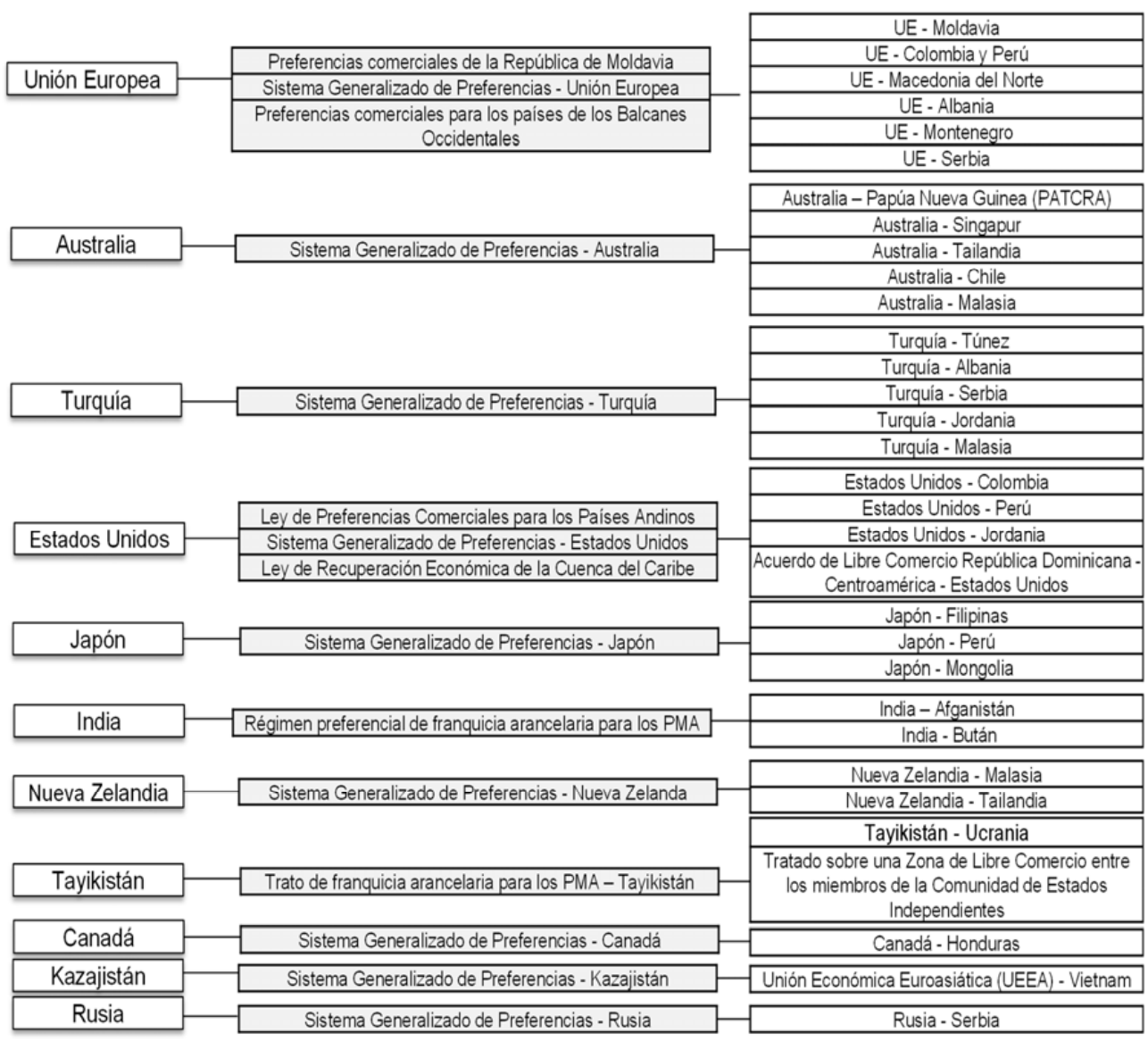

Fuente: elaboración propia con datos de la OMC (2019a).

Tal como se aprecia en los anteriores resultados, es clave denotar cómo en la actualidad de los treinta y cuatro SGP existentes se ha alcanzado un total de treinta y dos nuevos ALC que surgen de una iniciativa previa de tratado internacional en extinción y no de un proceso inédito de negociación entre dos países que buscan liberalizar conjuntamente su comercio exterior. En otras palabras, se ha generado una transición en cuyo caso, de los 34 SGP que se llegaron a firmar en algún momento, se han originado un total de treinta y dos nuevos ALC. Finalmente, en esta última parte de los resultados se hace imprescindible mostrar la influencia e impacto de los 32 nuevos ALC derivados de SGP (gráfico 5), sobre el número total de ALC en vigor registrados ante la OMC. 
Gráfico 5. Número de ALC derivados de SG dentro del sistema multilateral de comercio

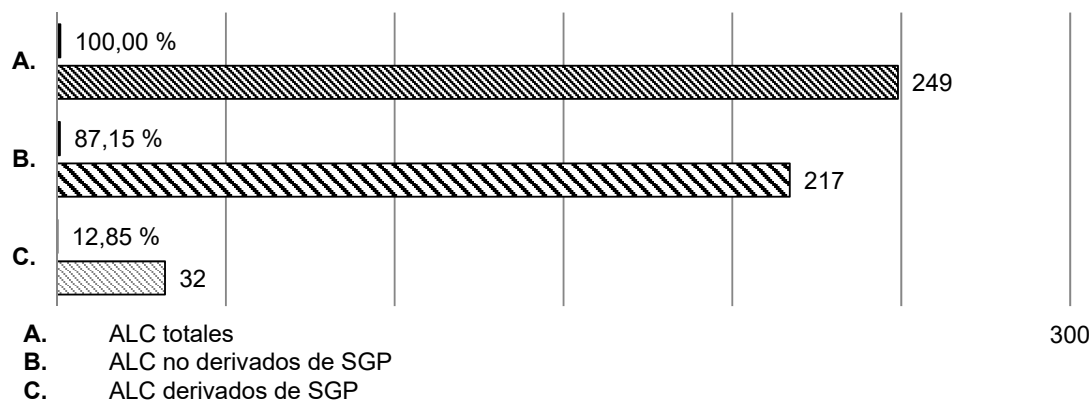

Fuente: elaboración propia a partir de la OMC (2019a, 2019b).

Tal y como se puede notar en el cierre de este apartado, los SGP que experimentaron una transición a ALC representan una cantidad sorprendente dentro del total de este tipo de tratados internacionales. Esta cantidad podría aumentar dado que aún están vigentes treita y un SGP que, de no ser renovados en el tiempo, podrían ejercer presión para negociar y firmar nuevos ALC con todos aquellos países menos adelantados que continúan recibiendo preferencias arancelarias unilaterales.

\section{CONCLUSIONES}

Se puede decir que de los treinta y cuatro SGP reportados ante la OMC desde la firma del GATT, incluyendo aquellos que no han sido prorrogados, se han generado un total de treinta y dos ALC. Lo anterior evidencia un notable cambio en los patrones de negociación de tratados comerciales entre gran parte de los países adelantados y países menos adelantados. Esta situación también demuestra que la cooperación, desde la perspectiva de este tipo de mecanismos, ha venido experimentando un preocupante declive que deja entrever un momento crítico para las relaciones internacionales. Todo esto se debe a que la unilateralidad de las preferencias tiende a desaparecer, lo que obliga a los países menos adelantados a asumir nuevos retos en cuanto a la liberalización de su comercio exterior.

Cabe agregar que los problemas de institucionalidad y voluntad política dentro de la OMC, tal como se ha venido apreciando dentro de las conferencias ministeriales y la misma Ronda Doha, claramente están incidiendo como un elemento adicional y categórico en la proliferación de ALC dentro del sistema multilateral de comercio. Esta situación deja en evidencia también otro momento crítico para el devenir de las relaciones internaciones dado que los países inmiscuidos en diferencias 
comerciales dentro de la OMC están invocando, de manera conveniente, las normas de los ALC dejando de lado las disposiciones del GATT y los demás textos jurídicos complementarios, motivo por el cual este tema debería discutirse de manera justa dentro de la OMC lo más pronto posible, dado que este asunto está terminando por socavar el sistema multilateral de comercio.

Adicionalmente, como futuras líneas de trabajo, es válido afirmar que la transición de SGP a ALC, señalada en este trabajo, puede responder también a otras causales asociadas, por ejemplo, al hecho de que tras la implementación de SGP los países menos adelantados han desarrollado dependencias substantivas que les obligan a llevar a cabo nuevos tratados aun cuando los países adelantados no desean renovar sus concesiones unilaterales con los SGP. Es decir, que la firma de ALC puede responder a los actuales problemas de consenso dentro del seno de la OMC, como se evidenció con la Ronda de Doha. De esta manera, empuja a los Estados a buscar otras formas de reglamentar temas comerciales puntuales. Asimismo, la firma de ALC puede responder también a la necesidad de los países menos adelantados por no perder su acceso al mercado en el país adelantado o en aquellos otorgantes en donde además el periodo de vida del SGP llega a su fin y tiende a no ser renovado debido al cambio, en la actualidad, de la política en cooperación internacional de muchos Estados.

\section{BIBLIOGRAFÍA}

Acharya, Sushant y Bengui, Julien (2018). Liquidity traps, capital flows. En: Journal of International Economics, vol. 114, n. ${ }^{\circ}$ 1, p. 276-298.

Baena, José (2018). Barreras arancelarias y no arancelarias como restricciones al comercio internacional. En: Revista Venezolana de Gerencia, vol. 23, n. 83, p. 543-562.

Baena, José (2019). La política de comercio exterior y las exportaciones colombianas. En: Revista de Economía Institucional, vol. 21, n. 41, p. 51-70.

Baena, José y Londoño, Abraham (2020). Tariff barriers and non-tariff barriers: appraising Colombia's protectionism. En: World Customs Journal, vol. 14, n. ${ }^{\circ} 1$, p. 71-94.

Baena, José y Fernández, Xavier (2016). Aproximaciones a la inserción de Colombia en el Sistema Multilateral de Comercio en 1995-2015. En: Análisis Político, vol. 29, n.o 87, p. 114-131.

Baena, José; Montoya, Anlly y Torres, Dayana (2017). La crisis económica mundial ¿La proliferación del proteccionismo como una causa-efecto? En: En-Contexto, vol. 5, n. 6, p. 185-207.

Bartels, Lorand (2003). The WTO enabling clause and positive conditionality in the European Community's GSP program. En: Journal of International Economic Law, vol. 6, n. 2, p. 507-532. 
Bearce, David (2003). Grasping the Commercial Institutional Peace. En: International Studies Quarterly, vol. 47, n. ${ }^{\circ}$ 3, p. 347-370.

Büthe, Tim y Milner, Helen (2008). The Politics of Foreign Direct Investment into Developing Countries: Increasing FDI through International Trade Agreements? En: American Journal of Political Science, vol. 52, n 4, vol. p. 741-762.

Cardona, Giovanny (2015). La Organización Mundial del Comercio y los TLC ċreinventando el Sistema Mundial de Comercio? Medellín: Editorial Ceipa, 206 p.

Cardona, Giovanny (2017). Comercio mundial: tendencias y estructura. Medellín: Editorial Ceipa, $398 \mathrm{p}$.

Cardona, Giovanny (2018). Integración económica y cooperación internacional: entre el multilateralismo, el regionalismo y la supranacionalidad. Medellín: Editorial Lasallista, 208 p.

Cassimatis, Anthony (2011). Developing Countries, Trade, and Human Rights: Free Trade Agreements, Development Needs, and the European Union's Generalized System of Preferences. En: Yong-Shik, Lee; Horlick; Won-Mog, Choi y Broude, Tomer (Eds.). Law and Development

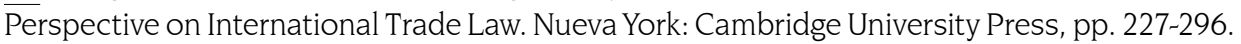

Crawford, Jo-Ann, y Laird, Sam (2000). Regional Trade Agreements and the WTO. https://www. nottingham.ac.uk/credit/documents/papers/00-03.pdf

Crawford, Jo-Ann, y Laird, Sam (2001). Regional trade agreements and the WTO. En: North American Journal of Economics and Finance, vol. 12, p. 193-211.

De la Reza, Germán (2015). Art. XXIV del GATT-OMC: la relación entre regionalismo y multilateralismo. En: Problemas del Desarrollo, vol. 46, n. ${ }^{\circ}$ 181, p. 185-204.

Del Río, Olga (2011). El proceso de investigación: etapas y planificación de la investigación. En: Vilches, Lorenzo (Coord.). La investigación en comunicación. Métodos y técnicas en la era digital. Barcelona: Gedisa, p. 67-93.

Diez, Alfredo (2001). Trato especial y diferenciado para países en desarrollo. En: Themis, vol. 43 , p. 249-259.

Dos Santos, Norma; Farias, Rogerio y da Cunha, Raphael (2005). Generalized System of Preferences in General Agreement on Tariffs and Trade/World Trade Organization: History and current issues. En: Journal of World Trade, vol. 39, n. ${ }^{\circ} 4$, p. 637-670.

Duarte, Lisbeth y González, Carlos (2014). Origen y evolución de la cooperación internacional para el Desarrollo. En: Panorama, vol. 8, n. 15, p. 117-131.

Estevadeordal, Antoni y Suominen, Kati (2009). The Sovereign Remedy? Trade Agreements in a Globalizing World. Nueva York: Oxford University Press, 267p.

Fernandez, Xavier (2009). Globalización económica, soberanía de los Estados y políticas sociales: funciones y retos del derecho internacional ante el "trilema" de la globalización. En: Sociedad Global, vol. 3, n. ${ }^{\circ}$ 1, p. 43-84.

García, Laura y Arévalo, Walter (2014). El trato especial y diferenciado en el sistema de solución de diferencias de la OMC como oportunidad para el ejercicio del derecho al desarrollo. En: Revista de Derecho, vol. 27, n. ${ }^{\circ}$ 1, p. 127-150. 
Giordano, Paolo; Mesquita, Mauricio y Quevedo, Fernando (2004). El tratamiento de las asimetrías en los acuerdos de integración regional. https://bit.ly/2ZbZrtm

Gómez, Marcelo (2006). Introducción a la metodología de la investigación científica. Córdoba: Brujas, $160 \mathrm{p}$.

Gurtner, Bruno (2010). The Financial and Economic Crisis and Developing Countries. En: International Development Policy, n. ${ }^{\circ}$ 1, p. 189-213.

Hartman, Stephen (2013). The WTO, the Doha Round Impasse, PTAs, and FTAs/RTAs. En: The International Trade Journal, vol. 27, n. ${ }^{\circ}$, p. 411-430.

Henckels, Caroline (2008). Overcoming Jurisdictional Isolationism at the WTO-FTA Nexus: A Potential Approach for the WTO. En: European Journal of International Law, vol. 19, n. 3 , p. 571-599.

Huici, Laura (2006). What kind of "generalized" systems of preferences? En: European Journal of Law and Economics, vol. 21, p. 267-283.

Jones, Vivian (2006). Generalized System of Preferences: Background and Renewal Debate. https:// apps.dtic.mil/dtic/tr/fulltext/u2/a487719.pdf

Kembayev, Zhenis (2009). Legal Aspects of the Regional Integration Processes in the Post-Soviet Area. Berlín: Springer, 217 p.

Kemp, Murray y Wan, Henry (1976). An elementary proposition concerning the formation of customs unions. En: Journal of International Economics, vol. 6, n. ํ 1, p. 95-97.

Kennedy, Kevin (2012). The Generalized System of Preferences After Four Decades: Conditionality and the Shrinking Margin of Preference. En: Michigan State International Law Review, vol. 20, n. ${ }^{\circ}$ 3, p. 521-668.

Kolb, Robert (Ed.) (2008). Encyclopedia of Business Ethics and Society. Londres: Sage, 2592 p.

Lacarte, Julio y Granados, Jaime (Eds.) (2004). Solución de controversias comerciales intergubernamentales: enfoques multilaterales y regionales. Buenos Aires: BID-Intal, 416 p.

Lynch, David (2010). Trade and Globalization: An Introduction to Regional Trade Agreements. Plymouth: Rowman y Littlefield Publishers, 346 p.

Mansfield, Edward y Milner, Helen (2012). Votes, Vetoes, and the Political Economy of International Trade Agreements. Nueva Jersey: Princeton University Press, 224 p.

Milewicz, Karolina; Hollway, James; Peacock, Claire y Snidal, Duncan (2018). Beyond Trade: The Expanding Scope of the Nontrade Agenda in Trade Agreements. En: Journal of Conflict Resolution, vol. 62, n. ${ }^{\circ}$ 4, p. 743-773.

Missios, Paul; Saggi, Kamal y Yildiz, Halis (2016). External trade diversion, exclusion incentives and the nature of preferential trade agreements. En: Journal of International Economics, vol. 99, p. 105-119.

Nayar, Shoba y Stanley, Mandy (Eds.) (2015). Qualitative Research Methodologies for Occupational Sciences and Therapy. Nueva York: Routledge, 254 p. 
OCDE. Organización para la Cooperación y el Desarrollo Económicos (2005). Preferential Trading Arrangements in Agricultural and Food Markets: The case of the European Union and the United States. París: OCDE, 184 p.

OMC. Organización Mundial del Comercio (2011). Informe sobre el comercio mundial 2011. https://bit.ly/2zPepdR

OMC. Organización Mundial del Comercio (2019). Arreglos comerciales preferenciales. https:// www.wto.org/spanish/tratop_s/region_s/rta_pta_s.htm

OMC. Organización Mundial del Comercio (2019a). Preferential Trade Arrangements. http:// ptadb.wto.org/default.aspx

OMC. Organización Mundial del Comercio (2019b). RTA Tracker. Recuperado de http://rtais.wto. org/UI/PublicMaintainRTAHome.aspx

Rivera-Batiz, Luis y Oliva, María (2003). International Trade: Theory, Strategies, and Evidence. Nueva York: Oxford University Press, 712p.

Robinson, William (2012). "The Great Recession" of 2008 and the Continuing Crisis: A Global Capitalism Perspective. En: International Review of Modern Sociology, vol. 38, n. ${ }^{\circ}$ 2, p. 169-198.

Rosales, Osvaldo (2009). Crisis internacional y oportunidades para la cooperación regional. https://bit.ly/368xtji

Ruiz, José Ignacio (2012). Teoría y práctica de la investigación cualitativa. Bilbao: Universidad de Deusto, $142 \mathrm{p}$.

Scott, James (2016). The International Politics of South-South Trade. En: Global Governance, vol. 22, n. 3 , p. 427-445.

Tassara, Carlo (2016). Cooperación internacional para el desarrollo: gobierno, economía y sociedad. Evolución de las políticas y escenarios futuros. Bogotá: Unisalle, 132 p.

Trejos, Alberto (2009). Instrumentos para la evaluación del impacto de acuerdos comerciales internacionales: aplicaciones para países pequeños en América Latina. Ciudad de México: Cepal, 66 p.

Trottier, Danielle (Ed.) (1987). Los programas de ajuste estructural y sectorial: alcances para la reactivación y desarrollo de la agricultura. San José: Instituto Interamericano de Cooperación para la Agricultura.

Urata, Shujiro (2002). Globalization and the growth in free trade agreements. En: Asia-Pacific Review, vol. 9, n. ${ }^{\circ}$ 1, p. 20-32.

Van der Berg, Hendrik (2017). International Economics: A Heterodox Approach. Nueva York: Routledge, 650p.

Vanegas, Juan Gabriel y Baena, José (2019). Virtual protectionism: Overview of MFN tariffs and bound tariffs in South America. En: Journal of International Studies, vol. 12, n. ${ }^{\circ}$ 4, p. 63-78.

Zahrnt, Valentin (2005). How regionalization can be a pillar of a more effective World Trade Organization. En: Journal of World Trade, vol. 39, n. ${ }^{\circ}$ 4, p. 671-699. 